\title{
Incentivo del Programa Cienciactiva a publicaciones científicas de estudiantes de medicina humana de una universidad pública del Perú
}

\section{Incentive of the Cienciactiva Program to scientific publications of medical students of a public university of Peru}

Correspondencia Andy Brian Campero-Espinoza andy.camperoe@gmail.com

Recibido: 23/11/2017

Aprobado: 06/12/2017

Citar como: Campero-Espinoza $A B$, Ccorahua-Rios MS, HuaracaParicahua SR, Atamari-Anahui N. Incentivo del Programa Cienciactiva a publicaciones cientificas de estudiantes de medicina humana de una universidad pública del Perú. Acta Med Peru. 2017;34(4):337-8
Andy Bryan Campero-Espinoza 1,2,a, Maycol Suker Ccorahua-Rios ${ }^{1,2, a}$, Sidahin Ranceth Huaraca-Paricahua ${ }^{1,2, a}$, Noé Atamari-Anahui ${ }^{1 b}$

1 Escuela Profesional de Medicina Humana, Universidad Nacional de San Antonio Abad del Cusco, Cusco, Perú.

2 ASOCIEMH CUSCO Asociación Científica de Estudiantes de Medicina Humana del Cusco, Universidad Nacional de San Antonio Abad del Cusco, Cusco, Perú.

a Estudiante de Medicina Humana, ${ }^{\mathrm{b}}$ Médico cirujano.

Hemos leído con interés el artículo de Huaylla-Zea et al. titulada "Investigación en salud y uso de recursos de canon minero en una universidad pública del Perú" ${ }^{[1]}$, la cual presenta un análisis sobre diferentes iniciativas que ha promovido la Universidad Nacional de San Agustín de Arequipa (UNSA) a través del convenio con Cienciactiva para impulsar la investigación.

En la formación médica la investigación constituye un eje principal; sin embargo, para realizarla los estudiantes se enfrentan a diversas limitaciones, dentro de las cuales está el aspecto económico. En nuestro país son pocas las universidades que brindan incentivos económicos a estudiantes que realizan y desean realizar investigación ${ }^{[2]}$. La Universidad Nacional de San Antonio Abad del Cusco (UNSAAC) en el marco del convenio con Cienciactiva realizó diferentes convocatorias para promover la investigación. Una de las convocatorias fue por publicación de artículos, cuya finalidad era brindar una subvención económica a las personas que publicaran artículos en revistas indizadas en bases de datos nacionales e internacionales, que tuvieran filiación de la UNSAAC ${ }^{[3]}$.

Con el fin de evaluar el efecto de este incentivo, se realizó una revisión en base los resultados emitidos por Cienciactiva mediante su plataforma de acceso público (http://www.cienciactiva.gob.pe/ unsaac/convocatorias/publicacion-de-articulos), con la finalidad de evaluar la cantidad de artículos, autores con su remuneración económica otorgada, el tipo de publicación y la base de datos a la cual pertenecían las publicaciones.

Los resultados muestran que las publicaciones en ciencias de la salud fueron $23,(24,7 \%)$ (Tabla 1), las cuales fueron publicadas en revistas indexadas en SciELO (61\%), SCOPUS (26\%), Dialnet (9\%) y Ebsco (4\%). Los tipos de publicaciones seleccionadas fueron: artículos originales (52\%), cartas al editor (26\%), reporte de caso (17\%) y un artículo original breve (4\%). En el $77,7 \%(14 / 18)$ de las publicaciones cuyos autores fueran estudiantes de medicina, se indicó pertenecer a la Asociación Científica de Estudiantes de Medicina Humana del Cusco (ASOCIEMH-Cusco), la cual está conformada por estudiantes de medicina de la UNSAAC. Además, el tercer autor con mayor número de publicaciones remuneradas de la convocatoria fue un estudiante de medicina humana. 
Tabla 1. Resultados de la primera convocatoria por publicación de artículos, convenio marco UNSAAC - Cienciactiva.

\begin{tabular}{lcccccc} 
& $\begin{array}{c}\text { Ciencias de la } \\
\text { Salud }\end{array}$ & $\begin{array}{c}\text { Total } \\
\text { (todas las áreas) }\end{array}$ & $\%$ & $\begin{array}{c}\text { Estudiantes } \\
\text { de Medicina }\end{array}$ & $\begin{array}{c}\text { Total (Ciencias } \\
\text { de la salud) }\end{array}$ & $\%$ \\
\hline Cantidad de publicaciones & 23 & 93 & 24,7 & 18 & 23 & 78,3 \\
Cantidad de autores & 6 & 35 & 17,1 & 5 & 6 & 83,3 \\
Subvención económica & S/ 47385 & S/ 278235 & 17,0 & S/ 35 235 & S/ 47385 & 74,4 \\
\hline
\end{tabular}

Se ha relacionado el hecho de pertenecer a una sociedad científica de estudiantes de medicina con una mayor producción científica ${ }^{[4]}$, ello podría explicar que la mayor parte de las publicaciones fueron realizadas por estudiantes que forman parte de la ASOCIEMH-Cusco.

En conclusión, consideramos importante el apoyo e incentivo a la producción científica que viene realizando Cienciactiva; esto incrementará el interés por parte de sus estudiantes y docentes y ayudará a generar investigaciones de calidad.

Financiamiento: Autofinanciado

Conflicto de intereses: Ninguno

\section{REFERENCIAS BIBLIOGRÁFICAS}

1. Huaylla-Zea C, Moreno-Loaiza M, Moreno-Loaiza O. Investigación en salud y uso de recursos de Canon minero en una universidad pública del Perú. Acta Med Peru. 2017;34(3):244-5.

2. Toro-Huamanchumo C, Arce-Villalobos L, Gonzales-Martínez J, Melgarejo-Castillo A, Taype-Rondán Á. Financiamiento de la investigación en pregrado en las facultades de medicina peruanas. Gaceta Sanitaria. 2017;31(6):541-2.

3. Cienciactiva-UNSAAC [Internet]. Lima: Concytec; 2017 [citado 13 de noviembre de 2017]. Disponible en: http://www.cienciactiva. gob.pe/unsaac/convocatorias/publicacion-de-articulos

4. Toro-Huamanchumo CJ, Failoc-Rojas VE, Díaz-Vélez C. Participación en sociedades científicas estudiantiles y en cursos extracurriculares de investigación, asociados a la producción científica de estudiantes de medicina humana: estudio preliminar. FEM. 2015;18(4):293-8.

\section{Ahora puede enviar sus artículos para Acta Médica Peruana en nuestro Open Journal System:}

\section{www.amp.cmp.org.pe}

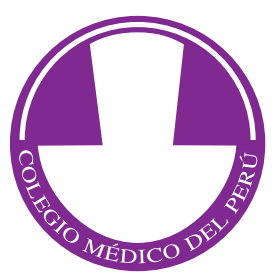

